\title{
A Practical Privacy Preserving E-Voting Scheme with Smart Card Using Blind Signature
}

\author{
V.K. Narendira Kumar \\ Assistant Professor, Department of Information Technology, \\ Gobi Arts \& Science College (Autonomous), \\ Gobichettipalayam - 638 453, Erode District, Tamil Nadu, India. \\ kumarmcagobi@yahoo.com \\ Dr. B. Srinivasan \\ Associate Professor, PG \& Research Department of Computer Science, \\ Gobi Arts \& Science College (Autonomous), \\ Gobichettipalayam - 638 453, Erode District, Tamil Nadu, India. \\ srinivasan_gasc@yahoo.com
}

\begin{abstract}
Voting is regarded as one of the most effective methods for individuals to express their opinions to select their democratic leader in the public elections. As the computing, communicating, and cryptographic techniques progress rapidly, increasing emphasis has been placed on developing electronic voting schemes capable of providing more efficient voting services than conventional paper-based voting methods. A receipt-free e-voting scheme based on the virtual voting booth that can be implemented with a smart card. Receipt-freeness is achieved by distributing the voting procedure between the voter and the smart card. By using smart cards to randomize part of content of the ballot, the voter cannot construct a receipt. The voter and the smart card jointly contribute randomness for the encryption of the ballot. To provide convenience to voters, sufficient voting facilities are supplied in sufficient public voting booths.
\end{abstract}

Index Terms - Electronic Voting, Smart Card, Election, Digital Signature, Internet, Security

\section{INTRODUCTION}

Elections are one of the most critical functions of the democracy. Not only do they provide for the orderly transfer of power, but they also cement citizen's trust and confidence in government when they operate as expected. Internet systems are among those being considered to replace older, less reliable systems. Election systems, however, must meet standards with regard to security, secrecy, equity, and many other criteria, making internet voting much more challenging than most electronic commerce or electronic government applications [1].

Internet voting systems can be grouped into three general categories: poll site, kiosk, and remote. Each of these categories define the location where the ballot is cast, which, in turn, defines the social science and technical hurdles that are associated with each type of system. Poll site internet voting offers the promise of greater convenience and efficiency than traditional voting systems in that voters could eventually cast their ballots from many polling places and the tallying process would be both fast and certain. Remote internet voting seeks to maximize the convenience and access of the voters by enabling them to cast ballots from virtually any location that is internet accessible. While the concept of voting from the home or work is attractive and offers significant benefits, it also provides substantial security risks and other concerns relative to civic culture. Without official control of the voting platform and physical environment, there are many possible ways for people to intervene to affect the voting process and the election results [8].

The remaining sections are organized as follows: Brief outline of Background of the E-voting is presented in section 2. E-Voting processes during the voting steps are mentioned in Section 3. The other phases of the e-voting identification system and smart card storage media are briefly explained in section 4 and 5. Implementations of the e-voting and Experimental results are given in Section 6 and 7. Finally, Section 8 describes the concluding remarks.

\section{BACKGROUND OF THE STUDY}

Electronic voting schemes without any security are unsuitable for being deployed in large-scale environments because a failure of a single voter would disrupt the entire voting. An electronic voting scheme based on the sender untraceable email system, which assumes that at least one mix is trust. Based on multiple key ciphers, a voting scheme, in which the voting authority can easily falsify the ballots. The security of their electronic voting schemes relies on the cooperation of the voters.

Proposed voting scheme is based on the homomorphic encryption technique, which can conceal the content of ballots, in a homomorphic encrypted ballot through the public channel, which is often implemented by a bulletin board. The encrypted ballots can be decrypted by any set of at least authorities. 
In the proposed a receipt-free and uncoercible electronic voting scheme is implemented with a smart card. The voter and the smart card jointly contribute randomness to the encryption of the ballot. Within the virtual voting booth, the voter interactively communicates with his smart card.

\section{A. Conventional Voting Systems}

Paper Ballots: Voters mark boxes next to the names of candidates or issue choices, and place them in a ballot box. The ballots are counted manually. Paper ballots are also widely used for absentee ballots. Their drawback is that counting is laborious and subject to human error.

Mechanical Lever Machines: Voters cast ballots by pulling down levers that correspond to each candidate or issue choice. Each lever has a mechanical counter that record the number of votes for that position. The machines prevent voting for more than one candidate. These machines are still widely used, but are no longer manufactured. Some versions do not produce an audit trail.

Punch Cards: Voters punch holes in computer readable ballot cards. Some systems use mechanical hole-punch devices for punching the holes while others provide the voter with pins to punch out the holes. The latter have been more subject to incomplete punches, resulting in more errors in reading the cards.

Optical Scan Devices: Voters record choices by filling in a rectangle, circle, or oval on the ballot. The ballots are read by running them through a computer scanner, which then records the vote [2].

Direct Recording Electronic (DRE) Devices: Specialpurpose or PC-based computers are used as voting machines. Voters use touch screens or push buttons to select choices, which are stored electronically in the memory of the machine. There are no paper ballots and no paper record independent of the electronic memory.

\section{B. Criteria for Election Systems}

Voting Principles: In general, the requirements for conventional, "paper based" voting also apply to electronic voting. These principals for democratic elections can be expected to be universal; of course, voting procedures may differ in many details.

Free Elections: The citizen must be able to use their voting rights without being coerced and without undue influence of a third party.

Secret Voting: No person must know the vote of another person.

Equal Voting Rights: Each vote must have the same weight. No vote must become invalid by predictable technical problems or must be lost on its way to the voting authority. Also, the right to vote must not be made dependent on factors other than those enumerated in the Law.

Audibility: The whole voting process must be transparent and reproducible.

Flexibility: The system should be configurable for many different election scenarios like different ballot question formats or multiple languages act and on a technical level compatible with multiple operation system platforms as well.

Uniqueness: No voter should be able to vote more than once.

Convenience: Election systems should not require extra skills to be usable and without unreasonable need for equipment [1].

\section{Traditional Paper Based Voting}

The electronic voting systems are based on the traditional paper based voting. Paper based voting is composed by a voting authority and the voters who are willing to express their whishes through the vote. The voting process as follows:

The voter is registered to vote by the voting authority. Usually a paper based identity is issued in the name of the voter.

In the day of the election, the voter's proceeds to the designated voting section, where it presents its voting identity.

The voting authority representative verifies the identity of the voter and gives permission for the voter to cast the vote. A paper with the voting options is given to the voter.

The voter proceeds to the secret ballots, where the voter writes in the official voting paper the whishes. The vote is cast into a sealed ballot.

After all votes are cast, the voting authority gathers all ballots and counts all votes. If a recount is necessary, the same ballots are recounted.

\section{Electronic Voting Systems}

An electronic voting system is an evolution of the paper based voting system. It comprises several forms of electronic devices such as electronic voting machines in kiosks, voting via internet, punch machine ballots with optical scanners, voting via email, etc. The same principles that are valid for the paper based voting are also valid for the electronic voting process.

\section{E. Electronic Voting using Smart Card}

In electronic voting systems the ballot box is remote and the voter uses computer networks to deliver the vote [5]. This voting system provides the voters with many benefits, such as the ability of issuing the vote from many different voting points and the possibility of getting the election result quickly. As elements of the general system architecture, smart cards have two essential functions:

Based in a set of keys and personal data stored in the cards, the voter is able to demonstrate their right to participate in the election. Similarly, the different management authorities and supervisors of the system have their own smart cards to guarantee the proper authentication [7]. Smart cards that are able to execute public key algorithms strongly guarantee the security the security of the operations and the privacy of the voters, facilitating the anonymity of the chosen option.

\section{Process ReQuirements DuRING Voting}


The average citizen cannot understand their internal requirements. Given that the people have a constitutional "right to designate the rulers of the state" it is not able that ownership and scrutiny of the casting, collecting and counting of votes has become a secret matter. In response to this, concerned private citizens have made use of the Freedom of Information to obtain as much relevant information as possible. People are getting more used to work with computers to do all sort of things to vote far from where they usually live, helping to reduce abstention rates. They may support arbitrary voting ballots and check their correct fulfillment during the voting process.

Authorization for Internet Ballot: The authorization for Internet balloting can be in various forms depending on the design of the Internet voting system as a whole. But any authorization must provide a way of linking the eventual vote cast using that registration to the registration record for that voter. So that it can be determined beyond a reasonable doubt that each Internet vote is associated with a registered voter in the proper district, and that at most one vote is counted for any voter. A server's response to the request for an Internet ballot will normally be to issue an authorization for Internet balloting to the voter who requested it. The authorization will be some combination of cryptographic keys, or PINs, or both, possibly accompanied by voting software.

Loss of Internet Ballot Authorization: Any system must be able to handle the voter's loss of, or failure to use, authorization for Internet balloting. If a voter loses Internet ballot authorization, or if that authorization for some reason fails to work to allow voting, then the voter can request a new Internet authorization. Before either such request is granted, the old authorization must be cancelled.

Voter Authenticates Their Self: Voters should be provided with an authentication code from the server that is combined with a Personal Identification Number (PIN) that will allow the voter to authenticate him/herself for the Internet voting system.

Voter Brings Internet Ballot to Screen: The screen on which the user views the ballot must be capable of rendering an image of the ballot in any of the languages and orthographies required by law for paper ballots. The application used for voting should not display or play any advertisement. Multi-page ballots should be easily navigable by voters, with no way to get lost or leave the balloting process except deliberately.

Voter makes choices: Voters should be able to point and click to make their voting selections. They should be able to navigate back and forth within the ballot to change selections freely until the moment when they click the final button that irrevocably transmits their ballot. Needs of voters with disabilities or impairments should be accommodated. The actual contents of the voter's votes on the client computer should be kept only in volatile memory.

Voter Casts Ballot: No vote must be transmitted before the voter clicks on a next-to-final button labeled, "Send Ballot". After clicking, the voter must be told that sending the ballot is final and must be asked to confirm voter intention to send the ballot by clicking a "Confirm" button. If the voter does not click the "Confirm" button, they should be able to return to the ballot to continue voting [5].

Ballot Transmitted to Vote Server: The ballot, along with a timestamp, voter's identification, precinct, and any other appropriate information, must be transmitted to the vote server in encrypted form to protect the privacy and integrity of the information.

Vote Server Receives Ballot: The ballot transaction is atomic. A ballot must be either wholly accepted, or wholly not accepted, by the vote server. There must be no middle ground. The vote server that receives a ballot should immediately check it to ensure that it is formatted correctly. If it is, the vote server should immediately store the ballot, still encrypted, on a permanent medium. The any subsequent power or equipment failure will not lose the ballot.

Vote Server Sends Feedback to Voter's Screen: Within a few seconds of receiving the ballot, the vote server should attempt to notify the voter of whether or not the vote was successfully accepted. If no feedback comes back to the voter's computer within a reasonable time, for any reason, then the voter is entitled to assume that the vote was not accepted, and may try again to vote.

Voter Can Ask For Confirmation after Casting of Vote: There must be a mechanism that voters can use to determine the status of their vote, whether or not it has been accepted and authenticated. After the voter has sent the ballot to the vote server, there must be no way for anyone, even the voter, to determine how they voted in any contest. In particular, there must be no way that a voter can prove to a third party how voter voted [6].

Votes Transmitted from Vote Server to Canvassing Machines: Internet voting systems must be capable of accurately tabulating the results and integrating the results with the server's primary voting system.

Authentication of votes and separation from voter identification: The election system server must be able to verify the authenticity of a ballot before the votes on the ballot are viewed or counted.

Canvassing Of Votes: The Internet voting system must be capable of accurately tabulating the results of all ballots cast. The canvass should only be conducted after the close of polls on Election Day.

Maintenance of Auditing Information: Decrypted ballots must retain in a secure format to allow for subsequent auditing and recount procedures [8].

\section{E-Voting IDENTIFICATION SYSTEM}

The system can be grouped in 3 different classes: PINBased or TAN-Based systems using smart cards for identification.

\section{A. Pin-Based Systems}

The voter is an identification user on the internet, after login the ballot sheet can be filled out and sent in, where the communication between the browser and the voting 
server is secured using cryptographic standards; it is obvious that anonymity cannot be guaranteed. Such systems can lower the transaction costs for elections drastically and in the case of dislocated voters be prerequisite for a fast election.

\section{B. TAN-Based Systems}

Number are issued and the election in usually possible by using the TAN in a Web browser. The connection between the voter and the Web server is also secured is also secured and the cryptographic key is issued by a Trust Center. The voter receives a random number as a receipt for casting the vote, which can be used to check whether the vote entered the tally correctly at a different Website.

\section{Smart Card-Based Systems}

Hence, neither PIN nor TAN based systems can be used for democratic elections, however, both are relatively easy to implement and can be used or a wide range of voting applications, where requirements for anonymity are less stringent or where anonymity is not a requirement at all. Systems using smart cards for digital signatures, which also enables the use of cryptographic methods is the choice for electronic voting [6].

\section{One-Stage Smart Card-Based Systems}

The algorithm assumes the use of a trust center for obtaining each party's public signature or crypto key. In its basic layout, the algorithm follows the registration ballot box approach. This algorithm has been implemented in various variations but all variants still maintained the basic problem: it is a one-phased algorithm, which means that both steps, identification and voting, are completed in one stage. When the administration of the registration and ballot box servers collude, it is possible to break the anonymity as well as to vote for voters that were entitled to vote but did not do so. The algorithm is secure on the application level, however, if the browser-based application provided by the registration step fraudulently stores the IP address for each blindly signed ballot sheet, and passes on this information to the ballot box, the clear-text ballot sheet after submission of m' can be linked to a voter later. Also temporary files could be used for this purpose. Hence, anonymity cannot be guaranteed if registration and vote submission are processed in one stage [9].

\section{E. Two-Stage Protocol}

The proposed algorithm strictly separates registration and vote submission stage. Registration Phase: The voter's credentials are checked and the voter receives a blindly signed election token, which is securely stored. Voting Phase: The voter uses the election token to obtain a ballot sheet and casts her vote.

\section{SMART CARD Storage MEdia}

As the algorithm uses a two-phase-protocol there is the need to temporarily store the token on a secure, anonymous medium.

\section{A. On the smart card used for the digital signature}

The advantage of storing the token on the voter's smart card is the protection from data loss as compared to conventional storage media and the protection from unauthorized access when the token is secured by a PIN from reading. The source code of the e-voting software can be made generally available and can be submitted to certification by an independent authority showing that neither the personal data nor the card number is accessed by the voting software, however, it seems doubtful whether this will be sufficient to gain public acceptance and since election token resides on the card between registration and election day, any other application accessing the card may read the personal information plus the token stored on the card thereby enabling a third party to trace the vote later [4].

\section{B. Storage Medium Similar To An Electronic Purse}

This variant solves the problems with serial number and clear text information discussed above: the voter uses a floppy disk or an USB-memory-key during the registration process and the token is saved on it. The implementation would be easy and would rely on general purpose infrastructure which is available off shelve.

\section{Smart Card Used for Digital Signature}

Another possibility would also be the use of a processor smart card, whose serial number is not registered or a storage card with in a minimum of processor functionality pure storage cards can be read and written to by general purpose card readers and in both variations there is no need for additional hardware. In both case, the card used for the digital signature is used only for identification purposes during the registration phase only and the token is stored on the second card. During the voting phase, only the storage card is used and anonymity can be preserved [3].

\section{IMPLEMENTATION OF E-VOTING SYSTEM}

Implementation is the process of converting a new system design into operation. Implementation is the key stage in achieving a successful new system as it involves a lot of upheaval in the system development process. This is carefully planned and controlled. A Primary implementation plan is prepared to schedule and manage many different activities that must be completed for a successful system implantation. The primary plan serves as a basis for checking the availability of resources for implementation activities.

Steps for Various Phases: Voting systems usually lead to a biased result that imparts the desired democracy. Unfortunately, these two problems become more difficult to solve when using e-voting schemes. Although many evoting schemes have been proposed to provide receiptfreeness to solve these problems, none is both secure and practical. In this research, an e-voting scheme that can solve or at least lessen the problems of bribe and coercion can be realized with current techniques. The techniques used for various phases are given below: 


\section{A. Ballot Generation Phase}

Step G1: Voter i goes to a VB that is convenient and safe for him, and authenticates himself to VB with his smart card $\mathrm{SC}_{\mathrm{i}}$, that has been activated by his characteristic.

Step G2: Voter i uses $\mathrm{SC}_{\mathrm{i}}$ to generate random numbers $r_{j}(\mathrm{j}$ $=1 \ldots \mathrm{L})$, and then uses $\mathrm{SC}_{\mathrm{i}}$ to compute $\mathrm{e}(\mathrm{j})=\left(\mathrm{g}^{\mathrm{rj}}\right.$, $\left.\mathrm{h}^{\mathrm{rj}} \mathrm{G}_{\mathrm{j}}\right)(\mathrm{j}=1 \ldots \mathrm{L})$. Next, Voter $i$ sends $\{\mathrm{e}(\mathrm{j}) \mid \mathrm{j}=$ $1 \ldots \mathrm{L}\}$ to $\mathrm{VB}$.

Step G3: VB generates random numbers $\mathrm{R}_{j}(\mathrm{j}=1 \ldots \mathrm{L})$ and computes $E(j)=\left(e_{1}(j) g^{R j}, e_{2}(j) h^{R j}\right)(j=1 \ldots L)$, where $\mathrm{e}(x)=\left(\mathrm{e}_{1}(x), \mathrm{e}_{2}(x)\right)$. VB generates random numbers $\mathrm{D}_{j}(\mathrm{j}=1 \ldots \mathrm{L})$, and computes $\left(\mathrm{a}_{\mathrm{j}}\right.$, $\left.b_{j}\right)=\left(g^{D j}, h^{D j}\right)(j=1 \ldots L)$, Next, VB generates random numbers $w_{j}$ and $\mathrm{N}_{\mathrm{j}}(\mathrm{j}=1 \ldots \mathrm{L})$, and computes $s_{j}=g^{w j} h^{R j N j}(j=1 \ldots L)$. Then, VB sends $\left\{E(j),\left(a_{\mathrm{j}}, b_{\mathrm{j}}\right) s_{\mathrm{j}} \mid \mathrm{j}=1 \ldots \mathrm{L}\right\}$ to Voter $i$.

\section{B. Ballot Casting Phase}

Step C1: Voter i uses $\mathrm{SC}_{\mathrm{i}}$ to generate random numbers, $\mathrm{d}_{\mathrm{j}}$, $\mathrm{k}_{\mathrm{j}}$ and $\mathrm{w}_{\mathrm{j}}{ }^{\prime}$, and compute $\mathrm{a}_{\mathrm{j}},=\left(\mathrm{x}_{\mathrm{j}}\right)^{\mathrm{dj}} \mathrm{g}^{\mathrm{kj}}(\mathrm{j}=1 \ldots, \mathrm{z}$ $-1, z+1, \ldots ., L), b_{j}=\left(y_{j}\right)^{d j} h^{k j}(j=1 \ldots, z-1, z$ $+1, \ldots, \mathrm{L}), \mathrm{a}_{\mathrm{z}}=\mathrm{g}^{w_{Z}+w_{z}{ }^{\prime}}$, and $\mathrm{b}_{\mathrm{z}}=\mathrm{h}^{w_{z}+w_{z}^{\prime}}$, where $\mathrm{z} \square,(1,2, \ldots, \mathrm{L}$,$\} is the number rep-$ resenting the option selected by Voter $\mathrm{i}$. Then, Voter $\mathrm{i}$ uses $\mathrm{SC}_{\mathrm{i}}$ to compute $\mathrm{B}=\mathrm{H}\left(\mathrm{ID}_{\mathrm{i}} \mathrm{x}, \mathrm{y}\right.$ $\left.\mathrm{x}_{1}, \ldots, \mathrm{x}_{\mathrm{L}}, \mathrm{y}_{1} \ldots \mathrm{y}_{\mathrm{L}}, \mathrm{a}_{1} \ldots, \mathrm{a}_{\mathrm{L}}, \mathrm{b}_{1}, \ldots, \mathrm{b}_{\mathrm{L}}\right)$ and $\mathrm{d}_{\mathrm{t}}=\mathrm{B}$, $\mathrm{d}_{\mathrm{j}}(\mathrm{j}=1 \ldots \mathrm{L})$.

Step C2: Voter i sends $\left\{B, d_{j} \mid j=1 . L\right\}$ to VB.

Step C3: VB sends $\left\{k_{J}=w_{j}+R_{j} d_{j} \mid j=1 \ldots L\right\}$ to Voter $i$.

Step C4: Voter $\mathrm{i}$ uses $\mathrm{SC}_{\mathrm{i}}$ to compute $\mathrm{R}_{\mathrm{z}}=\mathrm{w}_{z}{ }^{\prime}-\mathrm{k}_{\mathrm{z}} \mathrm{d}_{\mathrm{z}}+$ $K_{z}$, Voter i sends $\left\{E(z) B, d_{1}, d_{z}, \ldots, d_{L}, r_{1}, r_{2} \ldots\right.$, $\mathrm{r}_{\mathrm{L}}$ with signature $\}$ to $\mathrm{BB}$.

\section{Step for Tallying Phase}

Voting Authorities compute $(\mathrm{X}, \mathrm{Y})=\left(! \mathrm{Cx}_{\mathrm{i}}, ! \mathrm{Cy}_{\mathrm{i}}\right)$, where $\mathrm{x}_{\mathrm{i}}$ and $\mathrm{y}_{\mathrm{i}}$ denote the valid $\mathrm{x}$ and $\mathrm{y}$ of Voter $\mathrm{i}$, respectively. Next, Voting Authorities jointly (atleast $\mathrm{t}$ voting authorities) compute $\mathrm{W}=\frac{Y}{X^{s}}=G_{1}^{T_{l}} G_{2}^{T_{2}} \ldots G_{L}^{T_{L}}$. Then, Voting Authorities determine final tally $\mathrm{T}_{1}, \mathrm{~T}_{2}, \ldots$, $\mathrm{T}_{\mathrm{L}}$ from $\mathrm{W}$, and announce the Result. The system is developed using J2EE standards, implementation is much easier compared to other technologies. For implementation, there is a need for application server like Internet Explorer 6.0(or Higher Version). In the application server all the class files like jsp, HTML files will be placed in application folder.

\section{EXPERIMENTAL RESULTS}

The Voting Authority is responsible for controlling all voting servers which are used in the election. The access to resources in these servers is controlled using, besides physical security, digital certificates issued to the personnel responsible for running and administering them at the election period. The certification of the staff is set to expire when the election ends, so that no access is made after this period. The auditing of the system is made before, during and after the elections, by a team composed of the parties involved in the elections or someone appointed by them, the Voting Authority representative, IT security experts and representatives of the voters. The voting software essentially contains the following parts:

\section{The voting interface application \\ $>$ The security check software \\ $>$ The network client \\ $>$ The Blind Signature Protocol}

When connecting to the voting site, which is controlled by the Voting Authority, the user is requested to allow the voting program to be loaded and run on his computer, which will be used as the interface with the voting system during all the voting process. Since there is no way of telling which operating system the user has in the computer, a platform independent language must be used to build the software.

The first measure that will be performed by the voting program after it is loaded is to scan for security software in the voter's computer. This is in order to ensure that the vote will not be tracked or changed by a third party with malicious intentions. This process runs in the background, so the voter does not need to interact or be burdened by it. The program first starts a search in the voter's computer to seek installed antivirus, anti spyware/malware software and firewall programs. This assumption is mostly based on the fact that the majority of the users have Microsoft Windows installed in their computers, which is target of most of the threats nowadays seen.

Vote Casting: At this stage, the voter is ready to cast the vote. Protocols guarantee that the voter can vote anonymously and anonymous network secures the transmission of information in a public network. To achieve anonymity in the Internet, as well as in the connection between the voter's computer and the Voting Authority server, a network client is embedded in the voting program. The program initializes the network client which makes the network traffic anonymous on behalf of the voter.

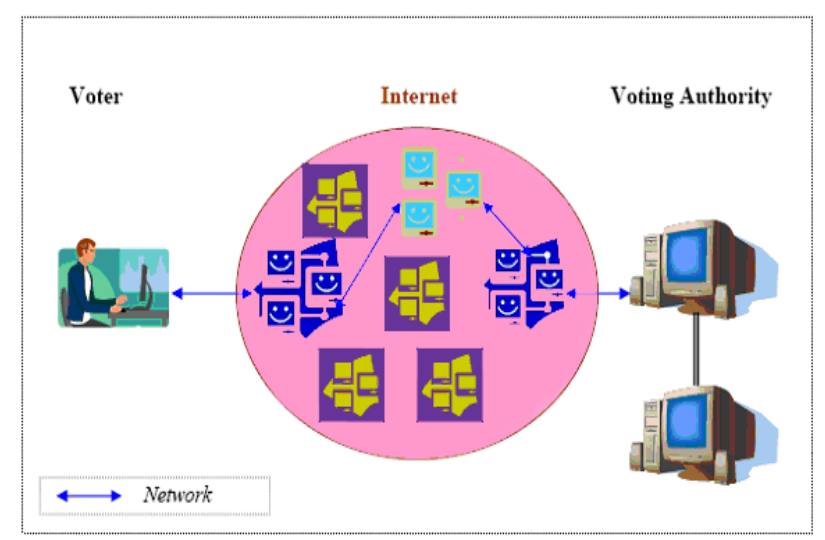

Fig. 1: Voter Network Architecture 
There are working clients already available to use for most operational systems, so it would be easy to implement a client inside the voting program. Next, the vote casting needs to be somehow made anonymous. This is where the Blind Signature protocol is used see Figure1.

The next step is the act of casting the vote. The voter now has the vote signed by the Voting Authority, and unbinds the vote. After that, the voter sends the vote through the anonymous network to the Voting Authority, and if everything is correct, the vote is stored in the voting database. The vote is then sent to the tallying server for totaling.

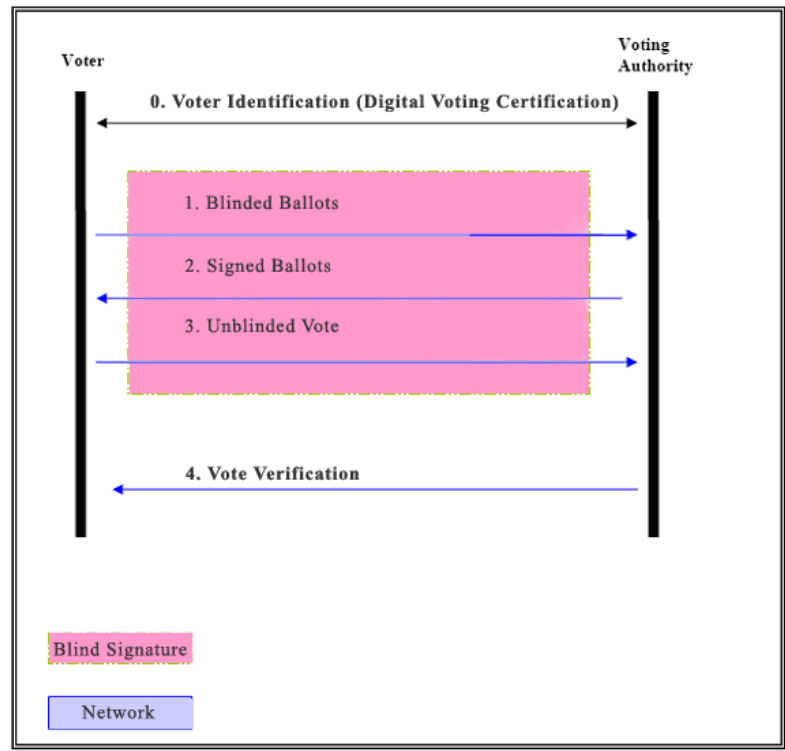

Fig. 2: Electronic Voting Process Using Digital Voting Identity, Blind Signatures and Network

The voter can verify his vote using the voting program, searching in the vote database or after the election in the voting list for the vote serial number to see if it was computed correctly. At the end of the election process all votes and their respective serial numbers are published to the public. The Figure 2 below summarizes the whole process.

Vote Scanning in Electronic Elections: There is no simple way to implement a paper voting system in a remote electronic voting through the Internet because the voter is not present in the voting premises to verify if the cast vote is correctly printed and inserted in the ballot. The Blind Signature algorithm can embed a serial number to the vote, but this implies that just the voter knows this number. To carry out a recount would then require that all voters check their votes. Also, the votes recorded electronically can be tampered in a much easier way than the paper ones. The proposed way to overcome this is to combine paper trial with image scanning to produce the same result as if the voter was present in the ballot room see Figure 3.

Registration Phase: In order to participate in election, a voter IDi, must first register to get his personal information e-mail account Eki, and identity information see Figure 4. Upon passing the verification of identification, the CA assigns a unique pair of public/private keys $\left(\mathrm{PK}_{\mathrm{i}} / \mathrm{SK}_{i}\right.$, ) containing in personal digital ID for the voter $I D$, to use in the e-mail software and web browser, then the CA places the voter's e-mail account and public key $P K$, in the "eligible registered voters list". CA notices the voter ID $_{\mathfrak{v}}$, to receive digital ID from secure SSL web site of CA. After the deadline of registration, CA transfers the "eligible registered voters list" to AC for the next phase.

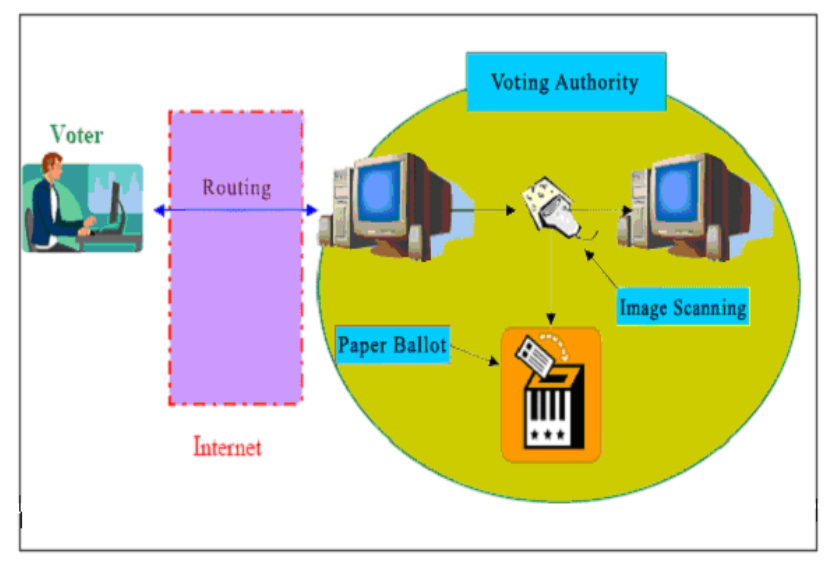

Fig. 3: Internet Voting with Image Scanning

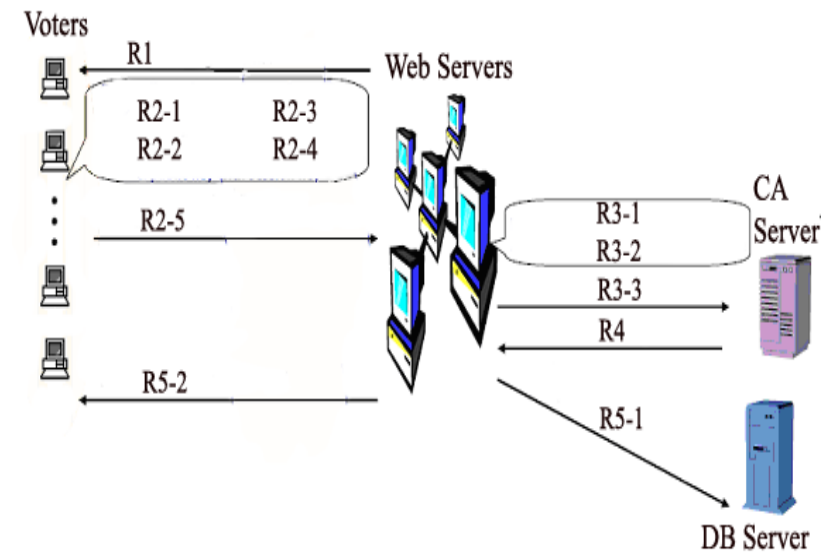

Fig. 4: Registration Stage

After setting up secure session

$\mathrm{R} 1=$ Download registration form

R2-1 = Fill out the registration form

R2-2= Generate private public key pair

R2-3 = Save private key in safe

R2-4 = Registration and public key with session key

R2-5 = Send encrypted message

R3-1 = Decrypted encrypted message

$\mathrm{R} 3-2=$ Generate request for PIN

R3-3= Send request for PIN

R4 = Issue PIN

R5-1 = Save registration information and PIN

R5-2 = Registration completed

Authentication Phase: The voter selects a digital pseudonym $V_{i}$ and a blind factor $r_{i}$, and uses the blinding function $R()$ to compute $\mathrm{X}_{i}=\mathrm{R}\left(V_{i}, r_{i}\right)$, Before mailing out, the voter uses his private key $S K_{i}$, to sign $X_{i}$, by the encryption function $E(), \mathrm{Y}_{i}=\mathrm{E}_{\mathrm{SKi}}\left(\mathrm{X}_{i}\right)$. And uses $\mathrm{AC}$ 's 
public key $P K_{A C}$ to encrypt the e-mail containing $Y_{i}$ and $X_{i} \mathrm{Z}_{i}=\mathrm{E}_{\mathrm{PK} \text { AC }}\left(\mathrm{Y}_{i}, \mathrm{X}_{i}\right)$. The voter uses his e-mail account $\mathrm{EM}_{i}$ to mail $Z_{i}$, to $\mathrm{AC}$ in order to apply for a "voting certificate". After AC has received the mail $Z_{i}$, AC uses its private key $S K_{A C}$ to decrypt $Z_{i},\left(\mathrm{Y}_{i}, \mathrm{X}_{i}\right)=\mathrm{D}_{\mathrm{SK} \text { AC }}\left(\mathrm{Z}_{i}\right)$. According to voter's e-mail account $\mathrm{EM}_{i}$, AC finds out the voter's public key $P K_{i}$ in the "eligible registered voter list", and uses it to verify the signature $Y_{i}, \mathrm{X}_{\mathrm{i}}=\mathrm{D}_{\mathrm{PKi}}\left(\mathrm{Y}_{\mathrm{i}}\right)$. AC uses its private key $S K_{A C}$ to sign $X_{i}$ blindly Before mailing $\mathrm{A}_{i}$ to the voter, $\mathrm{AC}$ uses the voter's public key $P K_{i}$ to encrypt the e-mail containing $\mathrm{A}_{i} \mathrm{~B}_{i}=\mathrm{E}_{\mathrm{PK} i}\left(\mathrm{~A}_{i}\right) . \mathrm{AC}$ replies $B_{i}$ to the voter, and marks on the "eligible registered voters list" according to voter e-mail account $E M_{i}$ in order to prevent from the double allocation of PIN (i.e to avoid assigning two PIN numbers for the single voter) 3 . After the voter received $\mathrm{B}_{i}$, he uses his private key $S K_{i}$ to decrypt the e-mail by the decryption function $D() \mathrm{A}_{i}=\mathrm{D}_{\mathrm{SK} i}\left(\mathrm{~B}_{i}\right) \quad$ In order to get the signature of pseudonym $V_{i}$, the voter removes the blind factor by the unblinding function $R^{-1}() S G_{i}=R^{-1}\left(A_{i}, r\right)$. In our scheme, in order to prevent AC making any mark on the ballot, voters link to the SSL secure web site of AC and down load blank ballots without any mark for the next voting phase see Figure 5.

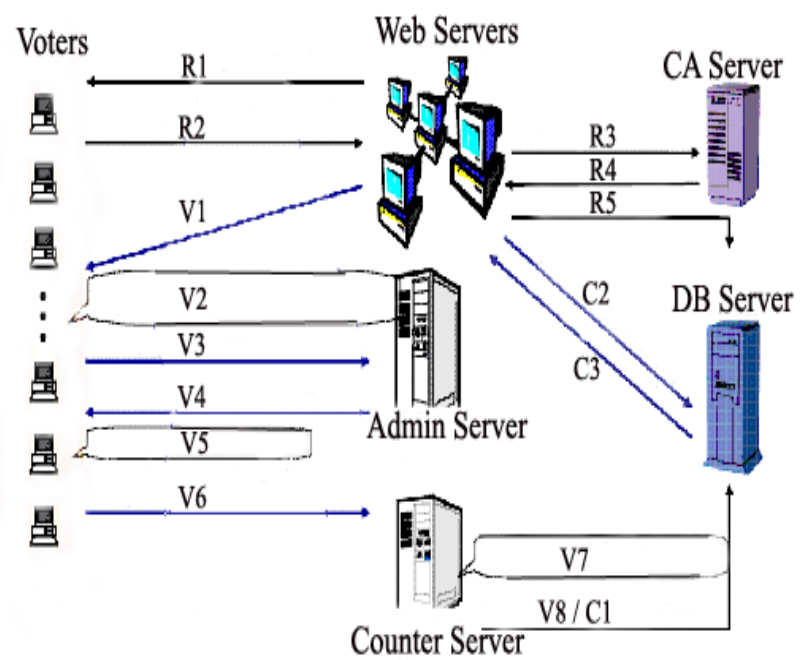

Fig. 5: Authentication Phase

$\mathrm{R} 1=$ Setup secure session, download registration form.

R2= Send encrypted public key and session key

$\mathrm{R} 3=$ Request personal information related to the voter

$\mathrm{R} 4=$ Send the requested information

$\mathrm{R} 5=$ Save the information

V1=Download voting applet

$\mathrm{V} 2=$ Encrypt the bollot with counter's public key

V3=Request blind signature

V4= Receive blind signature

$\mathrm{V} 5=$ Verify admin's blind signature

$\mathrm{V} 6=$ Send encrypted ballot and admin's digital signature

$\mathrm{V} 7=$ Verify decrypt ballot using counter's private key

$\mathrm{V} 8 / \mathrm{c} 1=$ Save all decrypted ballots

$\mathrm{C} 2=$ Send query for tallying

$\mathrm{C} 3=$ Receive the final result
Voting Phase: Before voting, the voter needs to randomize the ballot $\mathrm{M}^{\prime}$, and then encrypts it by using the secret sharing function $S(), T=R\left(M^{\prime}, r\right), C_{T C}=S_{T C}(T)$, $C_{S C}=S_{S C}(T)$. For the anonymity, the voter should login VC using the digital pseudonyms $V_{i}$ and its signature $\mathrm{SG}_{i}$. Meanwhile, the secret ballots $C_{T C} C_{S C}$ and $r$ are delivered to $\mathrm{VC}$. $\mathrm{VC}$ verifies the eligible identity by $\mathrm{v}_{i}=\mathrm{D}_{\mathrm{PK}}$ ${ }_{\mathrm{AC}}\left(S G_{i}\right)$. And VC records voter" in order avoid double voting. $V_{i}$ as a "voted to prevent the double voting. VC appends a serial number $S N_{J}$ and its signature $\mathrm{SG}_{S N}$ to each pair of $\left(\mathrm{C}_{\mathrm{TC}}, \mathrm{r}\right)$ and $\left(\mathrm{C}_{\mathrm{SC}}, \mathrm{r}\right)$ to guarantee that no facilities can duplicate or remove ballot privately, where $S G_{S N j}=E_{S K v c}\left(S N_{J}\right)$. VC sends $\left(S N_{J}, S G_{S N j}, C_{T C}, r\right)$ and $\left(S N_{J}, S G_{S N}, C s_{C}, r\right)$ to $T C$ and $\mathrm{SC}$ through the secure SSL file-transfer channel respectively see Figure 6.

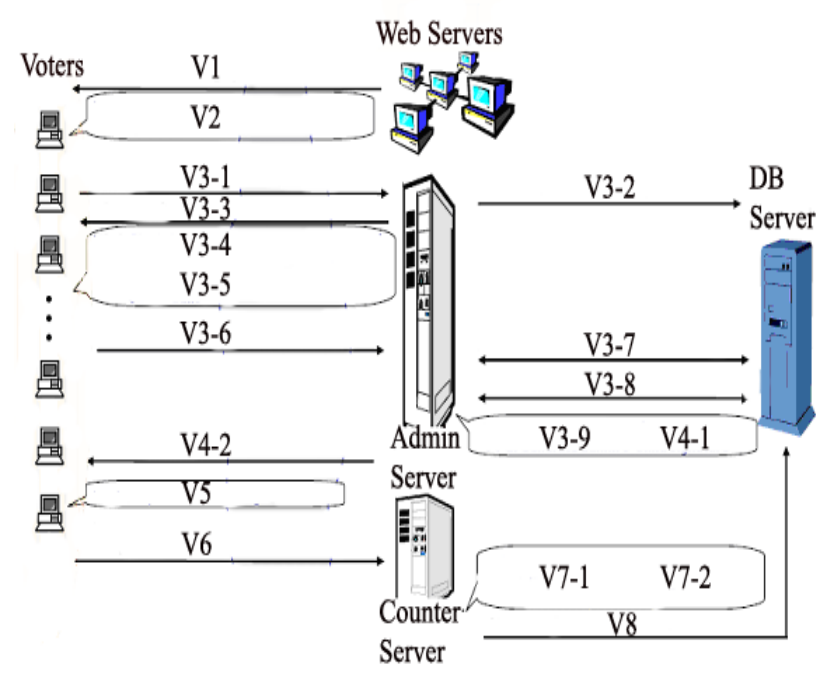

Fig. 6: Voting Stage

$\mathrm{V} 1=$ Voting webpage

V2 = Encrypt the ballot with counter's public key

V3-1 = Save

V3-2 = Request

V3-3 = Receive

V3-4 = Encrypted ballot used to received blinding factor

V3-5 $=$ Generate voter's signature on the ballot

V3-6 = Send voter's signature \& blinded information

V3-7 $=$ Request \& receive voter's certificate

V3-8 = Request \& receive voter's blinding factor

V3-9 = Verify voter's digital signature

V4-1 = Generate admin Blind Signature

V4-2 = Receive admin blind signature

$\mathrm{V} 5=$ Verify admin blind signature

V6 $=$ Send encrypted ballot and admin blind signature

V7-1 = Verify admin digital signature

V7-2 = Decrypt the ballot using counter's private key

V8 $=$ Save all decrypted ballots 


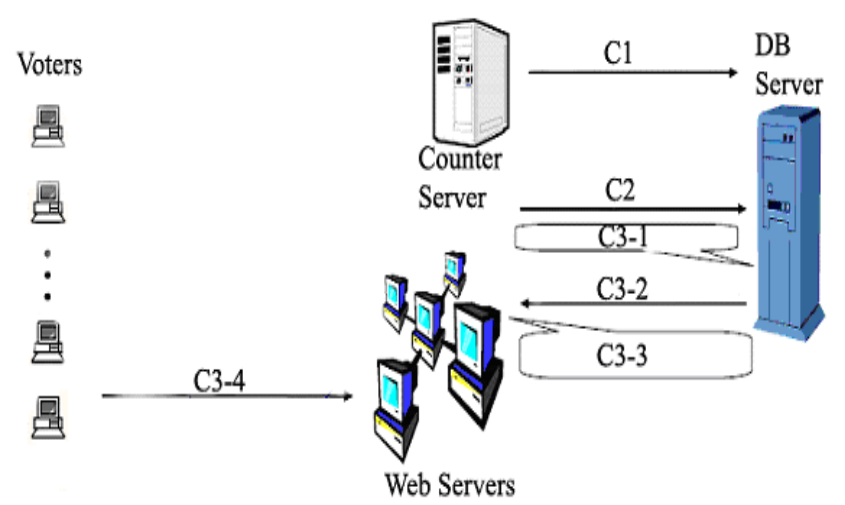

Fig. 7: Counting Stage

$\mathrm{C} 1=$ Save all decrypted ballots

$\mathrm{C} 2$ = Send query for tallying

C3-1 = Ballot counting

C3-2 = Receive the final result

C3-3 = Post the final result

C3-4 = Look up the final result

Counting Stage: At the end of the Election Day, the counting phase first implies that the tellers retrieve all ballots (in $D B_{B B}$ ) and all attendances (in $D B_{A S}$ ) from machines managed by the Controllers [6]. Then, the tellers can precede the results by first verifying that there are as many attendances as there are ballots. If there are more attendances than ballots or if one or more attendances are incorrect, it implies a problem either at the network layer level (lost packets), or a fraud of the Controllers. The good choice between the two can easily be made using logs if all transcripts are signed by all protagonists (since a signature provides non-repudiation and integrity). Then, for each ballot $B$, the tellers have first to decrypt the ballot to obtain $m=v+S=\operatorname{Decrypt}(B$; $e s k T)$. The next step consists in verifying the information produced by the voter. Again, if one is incorrect, this implies either that the Controllers have cheated or that another voter can cast instead of original voter. So fake votes are eliminated from counting process and the counting phase then begin see Figure 7.

\section{CONCLUSION}

This paper has highlighted the complexity of the deployment of smart cards operating under public key algorithms offers great advantages to guarantee both the voting anonymity and the voter's authentication. Since they are tamper-resistant, smart cards effectively protect personal keys of voters and the receipts generated after the voting. A new generation of smart cards, allow introducing in the card memory small applications, which support most of the needed cryptographic operations, maintaining in total secrecy the keys used for such operations. Although the small size of smart cards memory imposes certain limitations regarding the operations that can be carried out, adequate design and proper usage of existent tools permits to carry out complex and robust operations, which guarantee the global security of the system. The currently no global standards for electronic ballots and each system provide different solutions, which could be simplified if such a standard would be employed. With one common platform, it would be easier to concentrate efforts on developing and finding problems in electronic voting systems. Malicious code checking program must be installed in the voting software. Work is needed to test the case where the internet voting system is run in parallel with an Electron voting system, where voters can choose one of the systems to cast votes.

\section{REFERENCES}

[1] Alessandro Acquisti "Receipt-Free Homomorphic Elections and Write-In Ballots" - Technical Report 2004/105, International Association for Crypto Logic Research, May 2004, Page No. 56-68.

[2] Cohen, J., and M. Fischer. "A Robust And Verifiable Cryptographically Secure Election Scheme." Proceeding of the $26^{\text {th }}$ IEEE Symposium on Foundations of Computer Science, October 1985, Page No. 372-382.

[3] H. Nurmi, A. Salomaa, and L. Santean, "Secret Ballot Elections, In Computer Networks," Computers and Security, October, 2006. Page No. 96-108.

[4] M.bellare, A. Boldyreva, and J. Staddon. "Randomness Re-Use In Multi-Recipient Encryption Voting Schemes" Volume 25, 2003. Page No. 56-65.

[5] Neumann, Peter G. "Security Criteria for Electronic Voting" - $16^{\text {th }}$ National Computer Security Conference, September 2007. Page No. 210-219.

[6] Nurmi, H., et al. "Secret Ballot Elections in Computer Networks" - Computers \& Security, Vol. 10(2008): Page No. 553-560.

[7] O. Baudron, P. Fouque, D. Pointcheval, J. Stern, and G. Poupard, "Practical Multi-Candidate Election System" - ACM 20-th Symposium on Principle of Distributed Computing, PODC'01, 2001. Page No. 156-172.

[8] Peter Laud "Symmetric Encryption In Automatic Analyses For Confidentiality Electronic Voting" IEEE Symposium on Security and Privacy. Page No. 26-34.

[9] Saltman, Roy G. "Computerized Voting" - Chapter 5 In Advances In Computers, Vol. 32, Academic Press, 2009: Page No. 255-305.

\section{First Author Profile:}

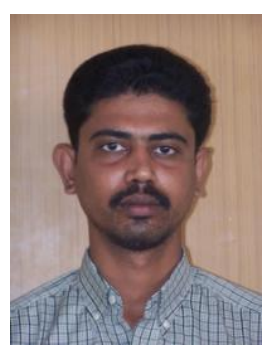

Mr. V.K. NARENDIRA KUMAR M.C.A., M.Phil., Assistant Professor, Department of Information Technology, Gobi Arts \& Science College (Autonomous), Gobichettipalayam - 638 453, Erode District, Tamil Nadu, India. He received his M.Phil Degree in Computer Science from Bharathiar University in 2007. He has author more than 21 
international journal article publications. He has authored or co-authored more than 60 technical papers and conference presentations. $\mathrm{He}$ is an editorial board member for several scientific international journals. His research interests are focused on Internet Security, Biometrics, Advanced Networking, Visual HumanComputer Interaction, and Multiple Biometrics Technologies.

\section{Second Author Profile:}

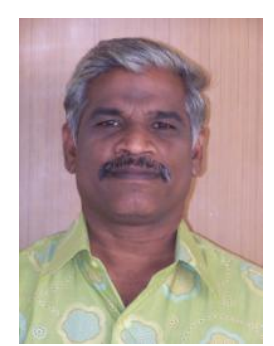

Dr. B. SRINIVASAN M.C.A., M.Phil., M.B.A., Ph.D., Associate Professor, PG \& Research Department of Computer Science, Gobi Arts \& Science College (Autonomous), Gobichettipalayam 638 453, Erode District, Tamil Nadu, India. He received his Ph.D. Degree in Computer Science from Vinayaka Missions University in 11.11.2010. He has authored or co-authored more than 70 technical papers and conference presentations. He is a reviewer for several scientific e-journals. His research interests include automated biometrics, computer networking, Internet security, and performance evaluation. 